\title{
PA-027 ADVERSE EVENT (AE) REPORTING FROM MALARIA MASS DRUG ADMINISTRATION (MDA) ROUNDS CONDUCTED IN SOUTHERN ZAMBIA
}

Victor Chalwe, John Miller. Ministry of Health, Zambia

\subsection{6/bmjgh-2016-000260.63}

Background The National Malaria Control Centre (NMCC) of the Ministry of Health in Zambia is conducting a large-scale mass drug administration (MDA) community randomised-controlled trial to evaluate the effectiveness of different MDA distribution strategies on reducing malaria parasitaemia. The trial involved two MDA strategies: MDA, where all eligible individuals were treated with dihydroartemisinin and piperaquine (DHAp), and focal MDA (fMDA), where all eligible individuals residing in a household with at least one positive member (rapid diagnostic test) were treated with DHAp. This provides an opportunity to document the extent to which potential safety issues are reported or adverse events occur given the level of exposure to treatments.

Methods Field teams comprised of community health workers, enumerators and adherence monitors, and supervised by facilitybased staff, received standardised training on the treatment campaign procedures, use of DHAp for eligible participants, adverse event monitoring, grading of events, and emergency and event handling procedures by grade. Adverse events were recorded on standard forms and in line with the national pharmacovigilance network recommendations. The principle aim of this data collection activity was to document and follow up on all adverse events (AEs) and serious adverse events (SAEs) occurring during the course of the MDA trial for individuals taking DHAp.

Results Four rounds of MDA were conducted over 2 years. During the first two intervention rounds, 280,638 participants were tested, 159,696 were treated with dihydroartemisininpiperaquine (DHAp) in 40 health catchment areas. During the second two intervention rounds, 261,814 participants took part. A total of 687 AEs $(0.13 \%$ of participants and $0.24 \%$ of treatments) were reported; four were recorded as serious adverse events (SAEs). The most common AE reported were gastrointestinal disturbances (diarrhoea, abdominal pain and nausea) 31.20\%; dizziness $19.8 \%$; vomiting $17.35 \%$; headache $16.03 \%$; and general body weakness at $11.37 \%$.

Conclusions During this large MDA trial, the use of DHAp for malaria treatment was generally safe and well tolerated. 